\title{
Rationale, Visionen und Grenzen der Immunonkologie: Checkpoint-Inhibition als neue Therapiesäule der Tumortherapie
}

\author{
Axel Hauschild ${ }^{a}$ Peter Mohr ${ }^{b}$ \\ ${ }^{a}$ Klinik für Dermatologie, Venerologie und Allergologie, Universitätsklinikum Schleswig-Holstein, Kiel, Deutschland; \\ b Hautklinik, Elbeklinikum Buxtehude, Deutschland
}

Die Entdeckung der Immun-Checkpoint-Inhibitoren hat sich bei der Entwicklung einer immunvermittelten, antitumoralen Behandlungsstrategie als wichtigste Therapieinnovation der letzten Jahre erwiesen [1]. Aus historischer Sicht hatte Paul Ehrlich schon Anfang des 20. Jahrhunderts die Vision, malignes Tumorwachstum über das körpereigene Immunsystem aufzuhalten: Er war davon überzeugt, dass sich das Prinzip einer aktiven Immunisierung in Analogie zur Impfung mit abgeschwächten Viren auch auf die Behandlung von Krebserkrankungen übertragen ließe [2]. In den späten 1950er-Jahren entwickelten Lewis Thomas sowie Frank Macfarlane Burnet das Konzept der Tumor-Immunüberwachung (cancer immune surveillance), wonach das adaptive Immunsystem über die Fähigkeiten verfügt, transformierte Zellen zu erkennen und zu eliminieren $[3,4]$.

\section{Entdeckung von spezifischen Tumorantigenen}

Zur Entdeckung tumorspezifischer Antigene führte die Forschung in den 1960er-Jahren anhand eines Mausmodells erste experimentelle Studien durch [5]. Zudem wurden Mäuse im Modellversuch dabei beobachtet, wie sie nach der Resektion eines chemisch induzierten Tumors ein erneut eingebrachtes Tumortransplantat des gleichen Tumors abstießen: Bei ihnen hatte das zelluläre Immunsystem offenbar eine protektive Immunantwort gegen bestimmte Tumormerkmale entwickelt - im Gegensatz zu anderen, genetisch identischen Mäusen, die am transplantierten Tumor verstarben [6]. Über den Einsatz von autologen, tumorzellspezifischen zytotoxischen CD8+ T-Lymphozyten, die bei Krebspatienten gewonnen wurden, sind Anfang der 1990er-Jahre auch die ersten humanen, tumorassoziierten Antigene (TAA) entdeckt worden - darunter z.B. das MAGE-1 (Melanoma Antigen-1) beim malignen Melanom [7].

Dennoch: Ungeachtet der TAA, die eine Tumorzelle exprimieren und die Erkennung durch das Immunsystem erleichtern können, gelingt es Tumoren, sich auch bei intakter Immunkompetenz zu manifestieren. Möglicherweise ist die Aktivierung des Immunsystems über TAA nicht ausreichend, um eine antitumorale Immunantwort zu induzieren: Tumorzellen können verschiedene Fluchtmechanismen (immune escape) nutzen, um der Immunüberwachung zu entkommen wie z.B. Antigenverlust, Verringerung der Immunogenität oder Schaffung eines protektiven Mikromilieus [8].

\section{T-Zell-vermittelte Langzeitremissionen}

Bei dem therapeutischen Bestreben, das antitumorale Potenzial des adaptiven Immunsystems zu nutzen bzw. die tumorinduzierten Resistenzmechanismen gegen das Immunsystem zu überwinden, stehen zytotoxische T-Lymphozyten schon länger im Mittelpunkt des wissenschaftlichen Interesses: Wie beispielweise im Rahmen einer allogenen Stammzelltransplantation und Donor-Lymphozyten-Infusionen zu beobachten war, konnten die antileukämischen Effekte dieser Therapieansätze auf die Reaktion von T- bzw. Natürlichen Killer (NK-)Zellen des Spenders gegen Tumorzellen des Empfängers im Sinne eines Graft-versus-Leukemia-Effekts zurückgeführt werden [9].

Zudem wurden in Gewebeproben diverser Tumorentitäten auffällig häufig CD4+ und CD8+ T-Lymphozyten aufgefunden: In vitro waren diese autologen tumorinfiltrierenden Lymphozyten (TILs) in der Lage, Tumorzellen zu lysieren [10]. Heute werden Verfahren evaluiert, autologe ex-vivo-expandierte TILs durch einen adaptiven Transfer therapeutisch nutzbar zu machen [10].

Weitere Evidenz dafür, dass autologe T-Zellen in der Lage sind, humane TAA zu erkennen und dass die Verstärkung ihrer Reaktivität bei einem Teil der Patienten eine langanhaltende Tumorremission auslösen kann, lieferten die klinischen Studienergebnisse der letzten Jahre, die insbesondere mit Immun-Checkpoint-Inhibitoren wie dem Anti-CTLA-4-Antikörper Ipilimumab sowie Anti-PD-1- bzw. PD-L1-Antikörpern erzielt wurden [9, 11-14].

\section{KARGER}

Fax +497614520714 
Abb. 1 Beispiele für co-inhibitorische Checkpoint-Moleküle (u.a. PD-1, CTLA-4, LAG-3), modifiziert nach Pardoll [17]. APC = antigenpresenting cell; $\mathrm{MHC}=$ major histocompatibility complex; TCR $=\mathrm{T}$ cell receptor.

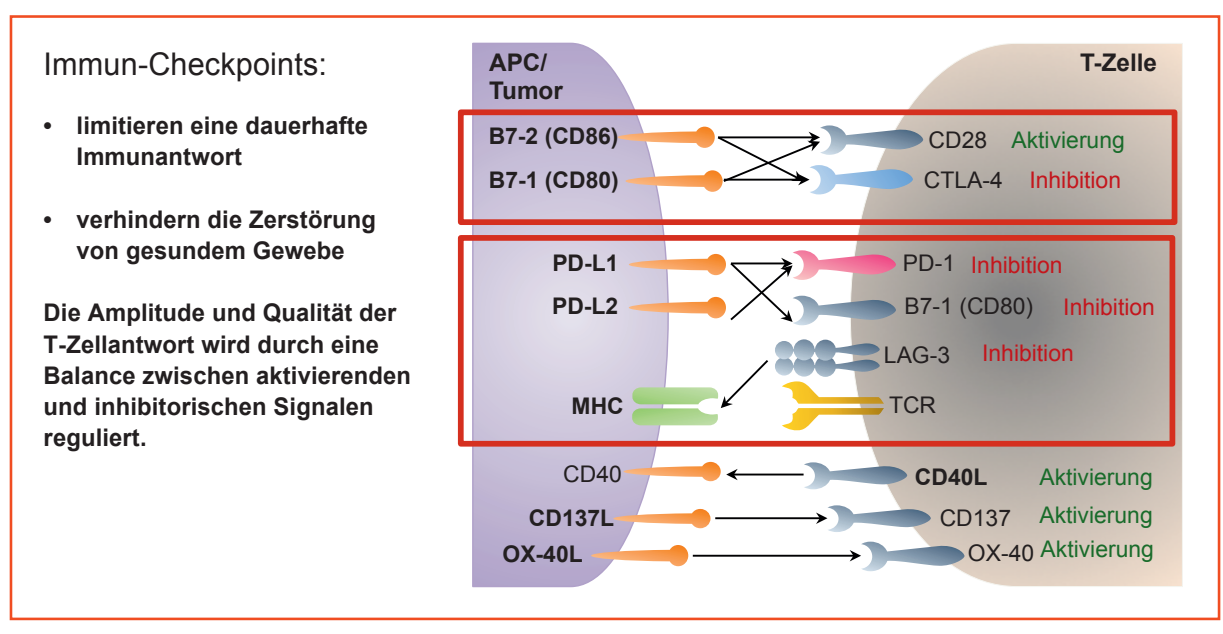

\section{Rationale für die Checkpoint-Inhibition}

CTLA-4 (Cytotoxic T-lymphocyte-associated antigen 4, auch: CD152) wurde in den späten 1980er-Jahren entdeckt und zunächst als Immunglobulin unbekannter Funktion charakterisiert, das in erster Linie auf CD4+ oder CD8+ T-Zellen exprimiert wird [15]. Heute weiß man, dass CTLA-4 zu einer wachsenden, funktionell zusammengehörigen Familie von Immun-Checkpoint-Rezeptoren gehört, die für die Modulierung der T-Zell-Antwort zuständig sind und zu der auch Liganden der B7-Familie (B7.1 (CD80) / B7.2 (CD86), PD-L1, PD-L2) sowie die TNF-Rezeptor-Familie (Tumornekrosefaktor) und deren Liganden zählen (Abb. 1). Ihnen allen ist die Eigenschaft gemein, als «zweites Signal» - sei es co-stimulatorisch oder co-inhibitorisch - die Intensität des «ersten Signals» zu modulieren, welches bei der Interaktion zwischen dem T-ZellRezeptor (TCR) sowie der Antigen-präsentierenden Zelle (APC) ausgelöst und an die T-Zelle adressiert wird. Ohne das zweite, aktivierende Signal induziert die Anbindung des Antigens eine TZell-Anergie, d.h. einen vorübergehenden Zustand der T-ZellInaktivität [16]. Tumorzellen können die negative Immunregulation über diese inhibitorischen Signalwege als Fluchtmechanismus zweckentfremden, um der antitumoralen Immunreaktion aktiv zu entkommen $[8,17]$.

\section{Brechung der peripheren Toleranz}

Mitte der 1990er-Jahre erkannte James P. Allison an seiner damaligen Wirkungsstätte, der Universität Berkeley, dass CTLA-4 in seiner physiologischen Funktion eine bremsende Wirkung auf TZellen ausüben muss und von der vollständigen Aktivierung einer Immunantwort abhält. Daraufhin entwickelte der Immunologe die Hypothese, dass sich die Inhibition der «T-Zell-Bremse» CTLA-4 als immunonkologischer Therapieansatz nutzen ließe. Der genaue Mechanismus der CTLA-4-mediierten T-Zell-Inhibition ist zwar noch Gegenstand laufender experimenteller Forschung, aber die zentrale Rolle als negatives Immunregulativ zeichnete sich damals schon im murinen, vollständigen Knock-out-Modell an Tieren ab: Der CTLA-4-Verlust führte zu einer massiven Lymphozytenproliferation und im Alter von 3-5 Wochen zum Tod [18]. Umgekehrt gelang es Krummel und Allison, über den Einsatz von CTLA4-Agonisten eine Hemmung der T-Zellproliferation und sinkende IL-2-Ausschüttung nachzuweisen [19].

Daraufhin wurden monoklonale Antikörper gegen murines CTLA-4 entwickelt und im Tiermodell angewandt: Die Gruppe von Allison konnte darunter nachweisen, dass sich die Immunreaktion unter der gezielten CTLA-4-Blockade sogar gegen etablierte Tumore verstärkte [20]. Zudem erwiesen sich die unerwünschten immuntoxischen Effekte unter der therapeutischen CTLA-4-Blockade im Gegensatz zum CTLA-4-Knock-out-Mausmodell als beherrschbar. Die CTLA-4-Blockade wurde in verschiedenen weiteren murinen Tumormodellen getestet, darunter an Karzinomen der Prostata und Brust oder auch Lymphomen, bevor im Jahr 2000 die ersten klinischen Versuche mit Ipilimumab, einem vollständig humanisierten Anti-CTLA-4-Antikörper, aufgenommen wurden [1]. Neben dem Potenzial, die Aktivität von Effektor-T-Zellen und die humorale Immunität zu verstärken, wurde die CTLA-4-Blockade unter Ipilimumab auch mit der möglichen Inhibition bzw. selektiven Funktionsbeeinträchtigung von regulatorischen T-Zellen (Tregs) in Verbindung gebracht, welche die periphere Toleranz gegenüber Selbstantigenen begünstigen können [21].

\section{Langzeitüberleben - «from Bench to Bedside»?}

Der Anti-CTLA-4-Antikörper Ipilimumab wurde 2011 als erster Immun-Checkpoint-Inhibitor beim metastasierten malignen Melanom zugelassen, nachdem er in einer randomisierten klinischen Studie einen signifikanten Überlebensvorteil gezeigt hatte [22]: In der zulassungsrelevanten Studie MDX010-20 erreichten vorbehandelte Patienten mit einem nicht resezierbaren bzw. fortgeschrittenen Melanom im Rahmen der Behandlung mit Ipilimumab ein medianes Gesamtüberleben (mOS) von 10,1 gegenüber 6,4 Monaten bei Patienten aus der Vergleichsgruppe, die eine Vakzinierung mit einem melanomspezifischen Glykoprotein 100 (gp100)-Vakzin erhielten (HR: 0,66; 95\% KI: 0,51-0,87; p = 0,0026). Zur Erinnerung: Vor der Ära der Checkpoint-Inhibitoren erreichten Melanom-Patienten im Stadium IV nach den bis dahin verfügbaren Standardtherapien unter Studienbedingungen ein medianes Gesamtüberleben von nur 6,2 Monaten [23]. 
Abb. 2. Langzeitverläufe mit Ipilimumab: gepoolte OS-Analyse (overall survival) inklusive EAP-Daten (Expanded Access Program) von 4846 Melanom-Patienten (modifiziert nach Schadendorf D et. al [24]).

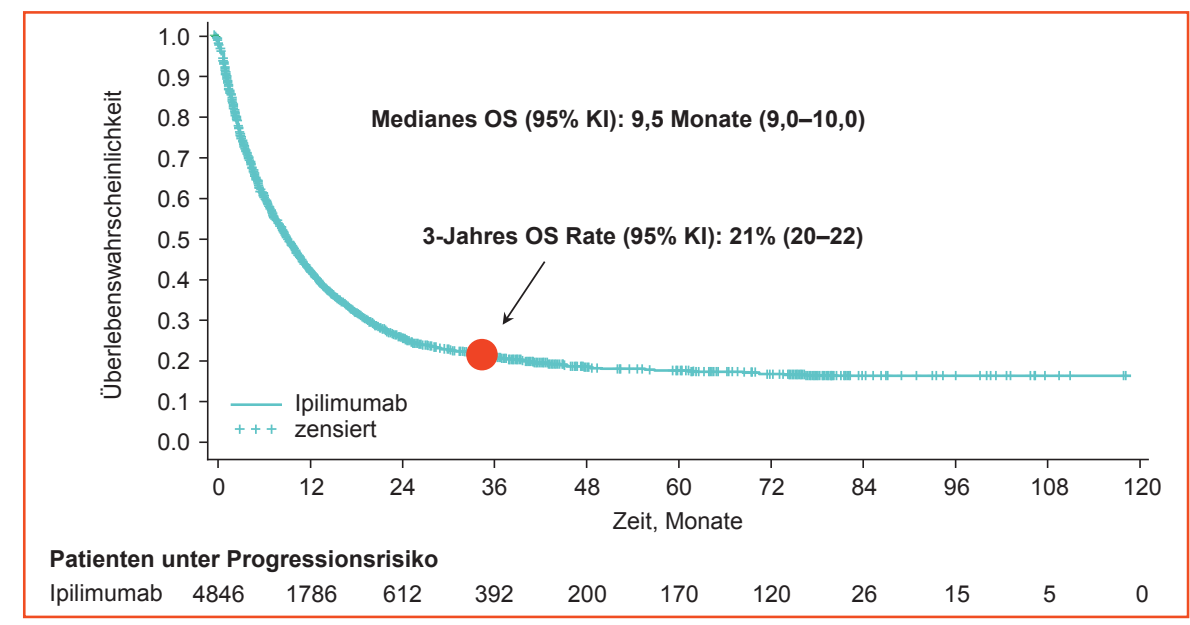

Eindrucksvoll war der Verlauf der Kaplan-Meier-Überlebenskurven des Ipilimumab-Arms im Vergleich zum Kontrollarm: In den Überlebenszeitanalysen nach 1 und 2 Jahren zeichnete sich ein bemerkenswerter Effekt von Ipilimumab auf die Überlebensraten ab [22]. 46\% der Patienten ( $\mathrm{n}=137)$, die mit Ipilimumab behandelt worden waren, erreichten ein 1-Jahres-Überleben (vs. 25\% im Vergleichsarm). Zwei Jahre ab Studieneintritt wiesen die Patienten des Ipilimumab-Arms noch eine Überlebensrate von $24 \%$ versus $14 \%$ in der mit gp100 allein behandelten Gruppe auf $(\mathrm{n}=136)$ [22]. Das Follow-up umfasste bis zu 55 Monate.

In einer gepoolten Überlebenszeitanalyse $(\mathrm{n}=4846)$ aus 12 retro-/prospektiven Phase-II/III-Studien $(\mathrm{n}=1861)$ sowie weiteren Daten aus dem «Expanded Access Program» (EAP, $\mathrm{n}=2985)$ zeichnete sich nach etwa 3 Jahren ein Plateaueffekt ab (Abb. 2): Ein Teil der Patienten lebte noch 10 Jahre nach der Behandlung mit Ipilimumab [24].

\section{Optimierung durch Kombination?}

Angesichts des überraschend lang anhaltenden Überlebensvorteils, den ein Teil der Patienten bereits erreichen konnte, wird intensiv nach weiteren Möglichkeiten gesucht, um das Gesamtüberleben zu verbessern [25-27]. Als vielversprechend gilt unter anderem die Kombination von CTLA-4- und PD-1-Checkpoint-Antikörpern. Bei PD-1 (programmed death 1, CD279) handelt es sich um einen weiteren negativ regulierenden Immun-Checkpoint, der Anfang der 1990er-Jahre in murinen T-Zell-Hybridomazellen entdeckt wurde, die dem programmierten Zelltod unterlagen [26]. Ähnlich wie CTLA-4 wird PD-1 auf aktivierten T-Lymphozyten und darüber hinaus auch auf NK- und B-Zellen exprimiert [27]. Während CTLA-4 aber vor allem für die frühe Aktivierungsphase von T-Zellen in lymphatischem Gewebe zuständig ist, besteht der PD-1-assoziierte Signalweg offenbar vor allem darin, die Aktivität von Effektor-T-Zellen bei einer Entzündungsreaktion in peripheren Organen bzw. Geweben einzuschränken und Autoimmunität vorzubeugen [28]. Bei der negativen Immunregulation spielt auch die Aufrecht- erhaltung der peripheren Toleranz (also auch Toleranz zu malignen Zellen im Tumormikromilieu) eine wichtige Rolle [29].

Eine therapeutische Blockade von PD-1 könnte demnach T-Zellen ansprechen, die ihre Priming-Phase im Lymphknotengewebe bereits hinter sich gebracht haben, somit schon Antigen-erfahren sind und im Rahmen einer Effektor-T-Zell-Reaktion in eine entzündliche Umgebung der Peripherie vorgestoßen sind. Folglich würde die T-Zell-Aktivierung auch weniger unspezifisch ausfallen als bei der CTLA-4-Blockade in der Frühphase der Immunantwort, womit möglicherweise auch die niedrigere Rate an immunvermittelten Nebenwirkungen zusammenhängt, die unter einer AntikörperBlockade von PD-1/PD-L1-Signalen beobachtet wurden [30-33].

Daneben nutzen Tumorzellen selbst die Expression von PD-L1/ PD-L2 bzw. die Interaktion von PD-1 mit PD-L1/PD-L2 dazu, der Immunüberwachung zu entkommen [34], sodass eine therapeutische Antikörper-Blockade von PD-1 und dessen Liganden diese Interaktionen beeinträchtigen und die T-Zell-Reaktivität erhöhen könnte [35].

\section{Checkpoint-Modifier in neue Therapiekonzepte integrieren}

Präklinisch konnte das synergistische Potenzial einer gemeinsamen Checkpoint-Blockade-Strategie, die 2 distinkte immunregulative Mechanismen betrifft, im murinen Tumormodell bzw. in vivo bereits bestätigt werden $[36,37]$. Neben dem gegen PD-1 gerichteten Checkpoint-Inhibitor Nivolumab (BMS-936558), der als erster Anti-PD-1-Antikörper in die klinische Entwicklung ging sowie Pembrolizumab (beide in den USA zugelassen und in Europa zur Zulassung eingereicht), werden zurzeit verschiedene andere, gegen PD-1 und PD-L1 gerichtete Antikörper (z.B. AMP224, MPDL3280A, BMS-936559, MEDI-4736) allein und in Kombination beim Melanom und vielen anderen Tumoren wie z.B. beim Lungenkarzinom, Nierenzellkarzinom oder Lymphom entwickelt. Beim fortgeschrittenen, nicht resezierbaren Melanom wird die kombinierte Checkpoint-Blockade mit Nivolumab plus Ipilimumab derzeit in einer klinischen Phase-III-Studie untersucht (CheckMate 067).

Zur Optimierung der immunvermittelten Tumorkontrolle und Steigerung der Ansprechraten mit einem Checkpoint-Inhibitor der 
ersten Generation sind auch alternative Kombinationspartner wie Antikörper-modulierende, co-inhibitorische bzw. co-stimulatorische Agonisten z.B. aus der TNF-Rezeptor-Familie von Interesse, die sich bereits in frühen Phasen der klinischen Entwicklung befinden (z.B. OX40, CD137, CD27) [38]. Weitere systemische Therapieansätze, die als potenzielle Kombinationspartner untersucht werden, reichen von einer Verstärkung der Checkpoint-Modulation mit Vakzinen oder Zytokinen über zielgerichtete (z.B. BRAF/ MEK-Inhibitoren beim Melanom), zytotoxische, physikalische und zelluläre Therapien. Fragestellungen wie die optimale Sequenzierung verschiedener Therapieansätze bedürfen einer weitergehenden, prospektiven Evaluation durch klinische Studien.

\section{Disclosure Statement}

Die Autoren waren Referenten beim «5. Interdisziplinären Expertenforum», das von Bristol-Myers Squibb unterstützt wurde.

\section{Kernpunkte}

- Die über viele Jahrzehnte vorangetriebene Grundlagenforschung zu immunregulatorischen Mechanismen und geeigneten molekularen wie zellulären «Targets» der Tumorgenese führte bereits zur erfolgreichen klinischen Anwendung einer Antikörper-basierten Blockade von Checkpoint-Molekülen.

- Die Immun-Checkpoint-Blockade zählt zu einem vielversprechenden systemischen Therapieansatz, um eine anhaltende Tumorremission beim Melanom und darüber hinaus auch bei weiteren Tumorentitäten zu induzieren: Sie hat das Potenzial, das Gesamtüberleben der Tumorpatienten zu verbessern.

- Der Anti-CTLA-4-Antikörper Ipilimumab hat sich zu einem Checkpoint-Inhibitor für verschiedene neue Kombinationsansätze z.B. mit weiteren immunmodulatorischen Antikörpern beim Melanom sowie in weiteren Indikationsbereichen entwickelt.

\section{Literatur}

1 Pardoll D, Drake C: Immunotherapy earns its spot in the ranks of cancer therapy. J Exp Med 2012;209:201-209.

2 Ehrlich P: On immunity with special reference to cell life: Croonian lecture; in Himmelweit B (ed): The Collected Papers of Paul Ehrlich, Vol. II: Immunology and Cancer Research. London, Pergammon, 1956.

3 Thomas L: Cellular and Humoral Aspects of the Hypersensitive States, ed Lawrence HS. New York, Hoeber-Harper, 1959.

4 Burnet FM: Cancer - a biological approach. 1. The process of control. Br Med J 1957;1:779-782.

5 Old LJ, Boyse LA: Immunology of experimental tumors. Annu Rev Med 1964;15:167.

6 Basombrio MA. Search for common antigenicities among twenty-five sarcomas induced by methylcholanthrene. Cancer Res 1970;30:2458-2462.

7 van der Bruggen P, Traversari C, Chomez P, et al.: A gene encoding an antigen recognized by cytolytic $\mathrm{T}$ lymphocytes on a human melanoma. Science 1991; 254:1643-1647.

8 Hanahan D, Weinberg RA: Hallmarks of cancer: the next generation. Cell 2011;144:646-674.

9 Heemskerk B, Kvistborg P, Schumacher TNM: The cancer antigenome. EMBO J 2013;32:194-203.

10 Balch CM, Riley LB, Bae YJ, Salmeron MA, Platsoucas $\mathrm{CD}$, von Eschenbach A, Itoh K: Patterns of human tumorinfiltrating lymphocytes in 120 human cancers. Arch Surg 1990;125:200-205.

11 Sznol M, Kluger HM, Hodi FS, et al. Survival and longterm follow-up of safety and response in patients (pts) with advanced melanoma (MEL) in a phase I trial of nivolumab (anti-PD-1;BMS-936558;ONO-4538). J Clin Oncol 2013;31(suppl):abstrCRA9006^.

12 Hamid O, Robert C, Daud A, et al.: Safety and tumor responses with lambrolizumab (Anti-PD-1) in melanoma. N Engl J Med 2013;369:134-144.

13 Robert C, Ribas A, Wolchok JD, et al: Antiprogrammed-death-receptor-1 treatment with pembrolizumab in ipilimumab-refractory advanced melanoma: a randomised dose-comparison cohort of a phase 1 trial. Lancet 2014;384:1109-1117.

14 Topalian SL, Drake CG, Pardoll DM: Targeting the PD-1/B7-H1(PD-L1) pathway to activate antitumor immunity. Curr Opin Immunol 2012;24:207-212.
5 Brunet JF, Denizot F, Luciana MF, et al.: A new member of the immunoglobin superfamily - CTLA-4. Nature 1987;328:267-270.

16 Harding FA, McArthur JG, Gross JA, et al.: CD28-mediated signalling costimulates murine $\mathrm{T}$ cells and prevents induction of anergy in T-cell clones. Nature 1992;356:607-609.

17 Pardoll DM: The blockade of immune checkpoints in cancer immunotherapy. Nat Rev Cancer 2012;12:252-264.

18 Tivol EA, Borriello F, Schweitzer AN, et al.: Loss of CTLA-4 leads to massive lymphoproliferation and fatal multiorgan tissue destruction, revealing a critical negative regulatory role of CTLA-4. Immunity 1995;3:541-547.

19 Krummel MF, Allison JP: CD28 and CTLA-4 have opposing effects on the response of $\mathrm{T}$ cells to stimulation. J Exp Med 1995;182:459-465.

20 Leach DR, Krummel MF, Allison JP: Enhancement of antitumor immunity by CTLA-4 blockade. Science 1996;271:1734-1736.

21 Peggs KS, Quezada SA, Chambers CA, et al.: Blockade of CTLA-4 on both effector and regulatory T cell compartments contributes to the antitumor activity of antiCTLA-4 antibodies. J Exp Med 2009;206:1717-1725.

22 Hodi FS, O’Day SJ, McDermott DF, et al.: Improved survival with ipilimumab in patients with metastatic melanoma. N Engl J Med 2010;363:711-723.

23 Korn EL, Liu PY, Lee SJ, et al.: Meta-analysis of phase II cooperative group trials in metastatic stage IV melanoma to determine progression-free and overall survival benchmarks for future phase II trials. J Clin Oncol 2008;26:527-534.

24 Schadendorf D, Hodi FS, Robert C, et al.: Pooled Analysis of Long-Term Survival Data From Phase II and Phase III Trials of Ipilimumab in Unresectable or Metastatic Melanoma. J Clin Oncol 2015 Feb 9. pii: JCO.2014.56.2736.

25 Maio M, Grob JJ, Aamdal S, et al.: Five-Year Survival Rates for Treatment-Naive Patients With Advanced Melanoma Who Received Ipilimumab Plus Dacarbazine in a Phase III Trial. J Clin Oncol 2015 10.1200/ JCO.2014.56.6018.

26 Ishida Y, Agata Y, Shibahara K, Honjo T: Induced expression of PD-1, a novel member of the immunoglob ulin gene superfamily, upon programmed cell death. EMBO J 1992;11:3887-3895.
27 Topalian SL, Drake CG, Pardoll DM.: Targeting the PD-1/B7-H1(PD-L1) pathway to activate antitumor immunity. Curr Opin Immunol 2012;24:207-212.28

28 Benson DM, Bakan CE, Mishra A, et al.: The PD-1/ PD-L1 axis modulates the natural killer cell versus multiple myeloma effect: a therapeutic target for CT011, a novel monoclonal anti-PD-1 antibody. Blood 2010;116:2286-2294.

29 Keir ME, Butte MJ, Freeman GJ, Sharpe AH: PD-1 and its ligands in tolerance and immunity. Annu Rev Immunol 2008;26:677-704.

30 Topalian SL, Hodi FS, Brahmer JR, et al.: Safety, activity, and immune correlates of anti-PD-1 antibody in cancer. N Engl J Med 2012;366:2443-2454.

31 Brahmer JR, Tykodi SS, Chow LQ, et al.: Safety and activity of anti-PD-L1 antibody in patients with advanced cancer. N Engl J Med 2012;366:2455-65.

32 Ibrahim R, Stewart R, Shalabi A: PD-L1 Blockade for Cancer Treatment: MEDI4736. Sem Oncol 2015;DOI:10.1053/j.semin oncol.2015.02.007.

33 Hamid O, Sosman J, Lawrence D, et al.: Clinical activity, safety, and biomarkers of MPDL3280A, an engineered PD-Ll antibody in patients with locally advanced or metastatic melanoma $(\mathrm{mM})$. J Clin Oncol 2013;31(suppl):abstr 9010.

34 Dong H, Strome SE, Salomao DR, et al.: Tumor-associated B7-H1 promotes T-cell apoptosis: a potential mechanism of immune evasion. Nat Med 2002;8:793-800.

35 Freeman GJ, Long AJ, Iwai Y, et al.: Engagement of the PD-1 immunoinhibitory receptor by a novel B7 family member leads to negative regulation of lymphocyte activation. J Exp Med 2000;192:1027-1034.

36 Curran MA, Montalvo W, Yagita H, Allison JP: PD-1 and CTLA-4 combination blockade expands infiltrating $\mathrm{T}$ cells and reduces regulatory $\mathrm{T}$ and myeloid cells within B16 melanoma tumors. Proc Natl Acad Sci USA 2010;107:4275-4280.

37 Das R, Verma R, Sznol M, et al.: Combination therapy with anti-CTLA-4 and anti-PD-1 leads to distinct immunologic changes in vivo. J Immunol 2015;194:950 959.

38 Bremer E: Targeting of the tumor necrosis factor receptor superfamily for cancer immunotherapy. ISRN Oncol 2013;2013:371854. 